\title{
A New Fuzzy Impulse Noise Detection Method for Colour Images
}

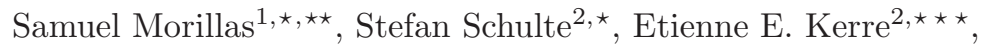 \\ and Guillermo Peris-Fajarnés ${ }^{1}$ \\ 1 Technical University of Valencia, E.P.S. de Gandia, Carretera Nazaret-Oliva s/n, \\ 46730 Grao de Gandia, Spain \\ smorillas@ieee.org \\ ${ }^{2}$ Ghent University, Department of Applied Mathematics and Computer Science, \\ Krijgslaan 281 - S9, 9000 Gent, Belgium \\ Stefan. Schulte@UGent. be
}

\begin{abstract}
This paper focuses on fuzzy image denoising techniques. In particular, we develop a new fuzzy impulse noise detection method. The main difference between the proposed method and other state-of-the-art methods is the usage of the colour components for the impulse noise detection method that are used in a more appropriate manner. The idea is to detect all noisy colour components by observing the similarity between (i) the neighbours in the same colour band and (ii) the colour components of the two other colour bands. Numerical and visual results illustrate that the proposed detection method can be used for an effective noise reduction method.
\end{abstract}

\section{Introduction}

Reduction of noise in digital images is one of the most basic image processing operations. Recently a lot of fuzzy based methods have shown to provide efficient image filtering $12 / 34567$. These fuzzy filters are mainly developed for images corrupted with fat-tailed noise like impulse noise. Although these filters are especially developed for greyscale images, they can be used to filter colour images by applying them on each colour component separately. This approach generally introduces many colour artefacts mainly on edge and texture elements. To overcome these problems several nonlinear vector-based approaches were successfully introduced [8,9,10,11,12,13,14,15,16,17,18,19]. Nevertheless all these vector-based methods have the same major drawbacks, i.e. (i) the higher the noise level is the lower the noise reduction capability is in comparison to the component-wise approaches and (ii) they tend to cluster the noise into a

\footnotetext{
* Corresponding author.

${ }^{\star \star}$ S. Morillas acknowledges the support of Spanish Ministry of Education, Science under program "Becas de Formación de Profesorado Universitario FPU".

$\star \star \star$ S. Schulte and E.E. Kerre acknowledge the support of Ghent University under the GOA-project 12.0515.03.
} 
larger array which makes it even more difficult to reduce. The reason for these disadvantages is that the vector-based approaches consider each pixel as a whole unit, while the noise can appear in only one of the three components.

In this paper another colour filtering method is proposed. As in most other applications we use the RGB colour space. The main difference between the proposed method and other state-of-the-art methods is the usage of the colour components for the impulse noise detection. The idea behind this detection phase is to detect all colour components which are dissimilar (i) to the neighbours in the same colour band and (ii) to the colour components of the two other colour bands. The proposed method illustrates the advantage of using the colour information in a more appropriate way to improve the noise reduction method. This work should also stimulate more research in the field of colour processing for image denoising.

The paper is organized as follows: in section 2 the new colour based impulse noise detection method is explained. A noise reduction method that uses the performed detection is described in section 3. Section 4 illustrates the performance of the proposed method in comparison to other state-of-the-art methods and the conclusions are finally drawn in section 5 .

\section{$2 \quad$ Fuzzy Impulse Noise Detection}

In this section a novel fuzzy impulse noise detection method for colour images is presented. In comparison to the vector-based approaches the proposed fuzzy noise detection method is performed in each colour component separately. This implies that a fuzzy membership degree (within $[0,1]$ ) in the fuzzy set noisefree will be assigned to each colour component of each pixel. When processing a colour, the proposed detection method examines two different relations between the central colour and its neighbouring colours to perform the detection: it is checked both (i) whether each colour component value is similar to the neighbours in the same colour band and (ii) whether the value differences in each colour band corresponds to the value differences in the other bands. In the following, the method is described in more detail.

Since we are using the RGB colour-space, the colour of the image pixel at position $i$ is denoted as the vector $\mathbf{F}_{i}$ which comprises its red $(\mathrm{R})$, green $(\mathrm{G})$, and blue (B) component, so $\mathbf{F}_{i}=\left(F_{i}^{R}, F_{i}^{G}, F_{i}^{B}\right)$. Let us consider the use of a sliding filter window of size $n \times n$, with $n=2 c+1$ and $c \in \mathbb{N}$, which should be centered at the pixel under processing. The colours within the filter window are indexed according to the scheme shown in Figure 1 for the $3 \times 3$ case. For larger window sizes the indexing will be performed in an analogous way. The colour pixel under processing is always represented by $\mathbf{F}_{0}=\left(F_{0}^{R}, F_{0}^{G}, F_{0}^{B}\right)$.

First, we compute the absolute value differences between the central pixel $\mathbf{F}_{0}$ and each colour neighbour as follows:

$$
\Delta F_{k}^{R}=\left|F_{0}^{R}-F_{k}^{R}\right|, \Delta F_{k}^{G}=\left|F_{0}^{G}-F_{k}^{G}\right|, \Delta F_{k}^{B}=\left|F_{0}^{B}-F_{k}^{B}\right|
$$




\begin{tabular}{|l|l|l|}
\hline 1 & 2 & 3 \\
\hline 4 & 0 & 5 \\
\hline 6 & 7 & 8 \\
\hline
\end{tabular}

Fig. 1. Vector index in the filter window

where $k=1, \ldots, n^{2}-1$ and $\Delta F_{k}^{R}, \Delta F_{k}^{G}, \Delta F_{k}^{B}$ denote the value difference with the colour at position $k$ in the $\mathrm{R}, \mathrm{G}$ and $\mathrm{B}$ component, respectively. Now, we want to check if these differences can be considered as small. Since small is a linguistic term, it can be represented as a fuzzy set [20]. Fuzzy sets in turn can be represented by a membership function. We compute the membership degree in the fuzzy set small $_{1}$ using the $1-S$-membership function [20] over the computed differences. This function is defined as follows

$$
1-S(x)= \begin{cases}1 & \text { if } x<\alpha_{1} \\ 1-2\left(\frac{x-\gamma_{1}}{\gamma_{1}-\alpha_{1}}\right)^{2} & \text { if } \alpha_{1}<x<\frac{\alpha_{1}+\gamma_{1}}{2} \\ 2\left(\frac{x-\alpha_{1}}{\gamma_{1}-\alpha_{1}}\right)^{2} & \text { if } \frac{\alpha_{1}+\gamma_{1}}{2}<x<\gamma_{1} \\ 0 & \text { if } x>\gamma_{1}\end{cases}
$$

where we have experimentally found that $\alpha_{1}=10$ and $\gamma_{1}=50$ receive satisfying results in terms of noise detection. In this case we denote $1-S$ by $S_{1}$, so that $S_{1}\left(\Delta F_{k}^{R}\right), S_{1}\left(\Delta F_{k}^{G}\right), S_{1}\left(\Delta F_{k}^{B}\right)$ denote the membership degrees in the fuzzy set small $_{1}$ of the computed differences with respect to the colour at position $k$. Now, we use the values $S_{1}\left(\Delta F_{k}^{R}\right), S_{1}\left(\Delta F_{k}^{G}\right), S_{1}\left(\Delta F_{k}^{B}\right)$ for $k=1, \ldots, n^{2}-1$ to decide whether the values $F_{0}^{R}, F_{0}^{G}$ and $F_{0}^{B}$ are similar to its component neighbours. The calculation of the membership degree in the fuzzy set noise-free is illustrated for the $\mathrm{R}$ component only but is performed in an analogous way for the $\mathrm{G}$ and $\mathrm{B}$ component. Because of the noise some of the neighbours could be corrupted with noise and therefore the values of $S_{1}\left(\Delta F_{k}^{R}\right)$ for $k=1, \ldots, n^{2}-1$ are sorted in descending order so that only the most relevant differences are considered. The value occupying the $j$-th position in the ordering is denoted by $S_{1}\left(\Delta F_{(j)}^{R}\right)$. Next, the similarity to the neighbour values is determined by checking that the value difference should be small with respect to, at least, a certain number $K$ of neighbours. The number $K$ of considered neighbours will be a parameter of the filter and its importance is discussed in section 4. So, we apply a fuzzy conjunction operator (fuzzy AND operation represented here by the triangular product t-norm 2122]) among the first $K$ ordered membership degrees in the fuzzy set $\operatorname{small}_{1}$. The conjunction is calculated as follows:

$$
\mu^{R}=\prod_{j=1}^{K} S_{1}\left(\Delta F_{(j)}^{R}\right),
$$

where $\mu^{R}$ denotes the degree of similarity of $F_{0}^{R}$ with respect to $K$ of its neighbours in the most favourable case. Notice that in the case that $F_{0}^{R}$ is noisy a low similarity degree $\mu^{R}$ should be expected. 
The next step of the detection process is to determine whether the observed differences in the $\mathrm{R}$ component of the processed colour corresponds to the same observations in the $\mathrm{G}$ and $\mathrm{B}$ component. We want to check if these differences agree at least for a certain number $K$ of neighbours. Then, for each neighbour we compute the absolute value of the difference between the membership degrees in the fuzzy set small $_{1}$ for the red and the green and for the red and the blue components, i.e. $\left|S_{1}\left(\Delta F_{k}^{R}\right)-S_{1}\left(\Delta F_{k}^{G}\right)\right|$ and $\left|S_{1}\left(\Delta F_{k}^{R}\right)-S_{1}\left(\Delta F_{k}^{B}\right)\right|$, where $k=$ $1, \ldots, n^{2}-1$, respectively. Now, in order to see if the computed differences are small we compute their fuzzy membership degrees in the fuzzy set small $_{2}$. A $1-S-$ membership function is also used but now we used $\alpha_{2}=0.10$ and $\gamma_{2}=0.25$, which also have been determined experimentally. In this case we denote the membership function as $S_{2}$. So we calculate

$$
\begin{aligned}
& \mu_{k}^{R G}=S_{2}\left(\left|S_{1}\left(\Delta F_{k}^{R}\right)-S_{1}\left(\Delta F_{k}^{G}\right)\right|\right), \\
& \mu_{k}^{R B}=S_{2}\left(\left|S_{1}\left(\Delta F_{k}^{R}\right)-S_{1}\left(\Delta F_{k}^{B}\right)\right|\right),
\end{aligned}
$$

where $\mu_{k}^{R G}$ and $\mu_{k}^{R B}$ denote the degree in which the observed difference in the red component is similar to the observed difference in the green and blue components with respect to the colour located at position $k$, respectively. Now, since we want to require that the differences are similar with respect to at least $K$ neighbours, the values of $\mu_{k}^{R G}$ and $\mu_{k}^{R B}$ are also sorted in descending order, where $\mu_{(j)}^{R G}$ and $\mu_{(j)}^{R B}$ denote the values ranked at the $j$-th position. Consequently, the joint similarity with respect to $K$ neighbours is computed as

$$
\mu^{R G}=\prod_{j=1}^{K} \mu_{(j)}^{R G}, \quad \mu^{R B}=\prod_{j=1}^{K} \mu_{(j)}^{R B},
$$

where $\mu^{R G}$ and $\mu^{G B}$ denote the degree in which the observed differences for the red component are similar to the observed differences in the green and blue components, respectively. Notice that if $F_{0}^{R}$ is noisy and $F_{0}^{G}$ and $F_{0}^{B}$ are not, then the observed differences can hardly be similar and therefore, low values of $\mu^{R G}$ and $\mu^{R B}$ are expected.

Finally, the membership degree in the fuzzy set noise-free for $F_{0}^{R}$ is computed using the following fuzzy rule 1

Fuzzy Rule 1. Defining the membership degree $N F_{F_{0}^{R}}$ for the red component $F_{0}^{R}$ in the fuzzy set noise-free:

IF $\mu^{R}$ is large $A N D \mu^{R G}$ is large $A N D \mu^{G}$ is large OR $\mu^{R}$ is large $A N D \mu^{R B}$ is large $A N D \mu^{B}$ is large

THEN the noise-free degree of $F_{0}^{R}$ is large

A colour component is considered as noise-free if (i) it is similar to some of its neighbour values $\left(\mu^{R}\right)$ and (ii) the observed differences with respect to some of its neighbours are similar to the observed differences in some of the other colour components $\left(\mu^{R G}\right.$ and $\left.\mu^{G B}\right)$. In addition, the degrees of similarity of the 

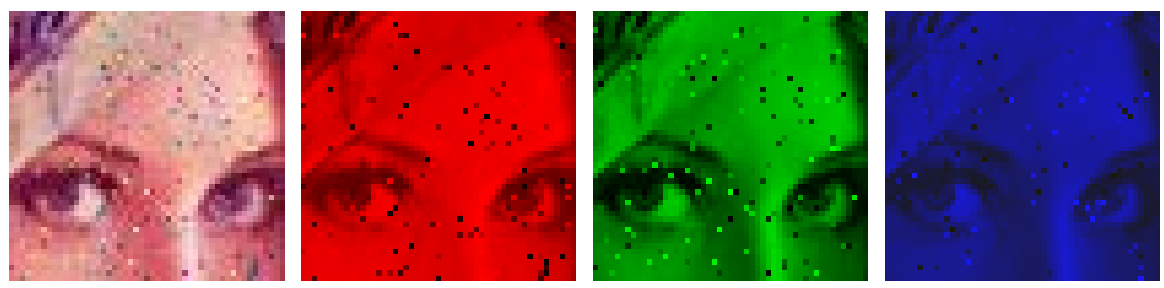

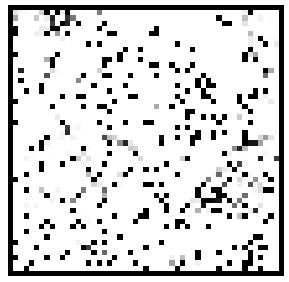

(a)

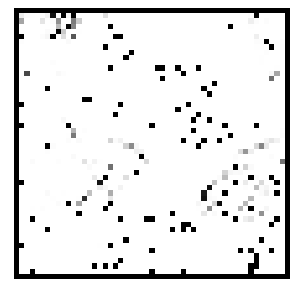

(b)



(c)

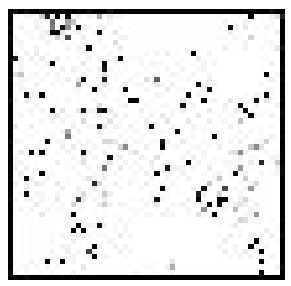

(d)

Fig. 2. An example of the proposed noise detection performance, with (a)-(d) Detail of the "Lena" image contaminated with $10 \%$ random-value impulse noise in each colour channel, and the computed noise-free degrees. Dark/white points indicate high/low noise-degree, respectively.

other components values with respect to their neighbour values, i.e. $\mu^{G}$ and $\mu^{B}$, are included so that a probably noisy component (with a low $\mu^{G}$ or $\mu^{B}$ value) can not be taken as a reference for the similarity between the observed differences. The fuzzy rule 1 contains four conjunctions and one disjunction. In fuzzy logic triangular norms and co-norms are used to represent conjunctions and disjunctions 2122 , respectively. Since we use the product triangular norm to represent the fuzzy AND (conjunction) operator and the probabilistic sum co-norm to represent the fuzzy OR (disjunction) operator the noise-free degree of $F_{0}^{R}$ which we denote as $N F_{F_{0}^{R}}$ is computed as follows:

$$
N F_{F_{0}^{R}}=\mu^{R} \mu^{R G} \mu^{G}+\mu^{R} \mu^{R B} \mu^{B}-\mu^{R} \mu^{R G} \mu^{G} \mu^{R} \mu^{R B} \mu^{B} .
$$

Notice that all the variables in the antecedent of the fuzzy rule 1 are already appropriate fuzzy values, so that no fuzzyfication is needed. Moreover, since we aim at computing a fuzzy noise-free degree, any defuzzyfication is neither needed.

Analogously to the calculation of the noise-free degree for the red component described above, we obtain the noise-free degrees of $F_{0}^{G}$ and $F_{0}^{B}$ denoted as $N F_{F_{0}^{G}}$ and $N F_{F_{0}^{B}}$ as follows

$$
\begin{aligned}
& N F_{F_{0}^{G}}=\mu^{G} \mu^{R G} \mu^{R}+\mu^{G} \mu^{G B} \mu^{B}-\mu^{G} \mu^{R G} \mu^{R} \mu^{G} \mu^{G B} \mu^{B}, \\
& N F_{F_{0}^{B}}=\mu^{B} \mu^{R B} \mu^{R}+\mu^{B} \mu^{G B} \mu^{G}-\mu^{B} \mu^{R B} \mu^{R} \mu^{B} \mu^{G B} \mu^{G} .
\end{aligned}
$$

In fuzzy logic, involutive negators are commonly used to represent negations. We use the standard negator $N_{s}(x)=1-x$, with $x \in[0,1]$ [21. By using this negation we can also derive the membership degree in the fuzzy set noise for 
each colour component, i.e. $N_{F_{0}^{R}}=1-N F_{F_{0}^{R}}$, where $N$ denotes the membership degree in the fuzzy set noise. An example of the proposed detection method is shown in Figure 2. Note that first/last rows/columns have not been processed in this example.

\section{Image Denoising Method}

Now we briefly explain an image denoising method that uses the fuzzy detection in section 2, The image is denoised so that (i) each colour component is smoothed according to its noisy degree and (ii) the colour information is used to estimate the output values. We propose to compute a weight for each colour component in order to calculate a weighted averaging to obtain the output. Now we illustrate the case of the $\mathrm{R}$ component but it is done in an analogous way for the $\mathrm{G}$ and $\mathrm{B}$ components. The denoised $\mathrm{R}$ component is obtained as follows

$$
\hat{F}_{0}^{R}=\frac{\sum_{k=0}^{n^{2}-1} W_{F_{k}^{R}} F_{k}^{R}}{\sum_{k=0}^{n^{2}-1} W_{F_{k}^{R}}}
$$

where $\hat{F}_{0}^{R}$ denotes the estimated value for the $\mathrm{R}$ component, $F_{k}^{R}, k=0, \ldots, n^{2}-1$ denote the $\mathrm{R}$ component values in the filter window and $W_{F_{k}^{R}}$ are their respective weights. The weight of the component being processed $W_{F_{0}^{R}}^{k}$ is set proportionally to its noise-free degree $N F_{F_{0}^{R}}$ so that it will be less weighted, and therefore more smoothed, if its noise-free degree is lower. The weight of the neighbour components is set inversely proportional to the noise-free degree of the component being denoised $N F_{F_{0}^{R}}$. Therefore, the neighbours are more weighted as $N F_{F_{0}^{R}}$ is lower. In addition, in order to take into account the colour information, we will weigh more those components $F_{k}^{R}$ for which it can be observed that $F_{k}^{G}$ is similar to $F_{0}^{G}$ or that $F_{k}^{B}$ is similar to $F_{0}^{B}$. The underlying reasoning is that if two colours have similar $\mathrm{G}$ or $\mathrm{B}$ components then it is observed that the $\mathrm{R}$ component is also similar. Notice that in a extremely noisy situation it may happen that $W_{F_{k}^{R}}=0, \forall k$ and then the weighted average cannot be performed. In such situations we perform a weighted vector median (WVM) operation, instead. In the WVM the weight of each vector should be set according to the vector noisefree degree which is computed as the conjunction of the noise-free degree of its RGB components.

\section{Parameter Setting and Experimental Results}

In this section we evaluate the performance of the proposed method and compare it with the performance of other methods. We use the Peak Signal-to-Noise Ratio (PSNR) 9] as objective measure to evaluate the quality of the filtered images.

In order to set the $K$ parameter of the filter we have taken different images and we have contaminated them with random-value impulse noise varying its 
percentage from $1 \%$ to $50 \%$ in each colour component. We have computed the performance (PSNR) achieved by the proposed filter using a $3 \times 3$ filter window for all possible values of $K \in\{1, \ldots, 8\}$. The obtained results seem to indicate that the most appropriate values of the $K$ parameter are $K=2,3$. When the images are contaminated with low-medium percentages of noise, setting $K=$ 2,3 makes the filter able to properly detect and reduce impulse noise while preserving noise-free image areas, specially edges and textures. However, when the percentage of noise is high it is observed that some clusters of similar noisy pixels may occasionally appear in the noisy images. Using a value of $K=2,3$ may not be able to reduce clusters of noisy pixels larger than or equal to 3 or 4 pixels. This problem can be solved by using a larger value for $K$ (maybe $K=4,5$ ), but in this case the performance for low densities of noise would be far from optimal because the detail-preserving ability is not so good as it is for lower values of $K$. Instead of this, we propose to perform a filtering based on a twostep approach. In the first step the noisy image is filtered using a $3 \times 3$ window and $K=2$. In this step, isolated noisy pixels are reduced while uncorrupted edges and details are properly preserved. In the second step, the image is now filtered using a $5 \times 5$ window and $K=5$. This step is intended to remove possible clusters of noisy pixels that may still remain in the image.

In the following the performance of the proposed filtering procedure, which we will entitle as impulse noise reduction method (INR), is compared to the performance of other state-of-the-art filters. The set of filters chosen for the comparison includes some filters for grayscale images applied in a component wise way (UF [19] and FRINRM [6]) and some colour image filters (VMF [1], PGSF [15], FISF [16] and FIDRMC [7). Notice that some of the mentioned filters are also based on fuzzy concepts (FRINRM, FISF and FIDRMC). We have used the three well-known images Baboon, Boats and Parrots for the tests. These images have been corrupted with different percentages of random-value impulse noise in each colour channel. We have used the following percentages: $5 \%, 10 \%, 15 \%, 20 \%, 25 \%, 30 \%, 40 \%$.

Since the proposed method uses a two-step procedure we have also filtered the test-images with the proposed filters using an analogous two-step design. The first step uses a $3 \times 3$ filter window where we used the (optimal) parameter setting suggested in the corresponding works. After the first step we have performed a second step where we use a $5 \times 5$ window size and where the corresponding (optimal) parameters are changed accordingly to the number of pixels in the window. In Tables 12 we have illustrated the PSNR performance achieved by all filters. The performance of the state-of-the-art methods included in the tables corresponds with the best performance achieved by the first or second step. Numbers followed by a $*$ indicate that the best performance is achieved in the first step. If no $*$ is used then the best performance is achieved by the second step. Some outputs of the filters for visual comparison are included in Figure 3 using a detail of the Parrots image corrupted by $15 \%$ of noise in each colour channel. From these results we can make the following conclusions: 
Table 1. Some experimental results for comparison in terms of PSNR using the Baboon image corrupted with different densities of random-value impulse noise

\begin{tabular}{|c|l|l|l|l|l|l|l|}
\hline Filter & $5 \%$ & $10 \%$ & $15 \%$ & $20 \%$ & $25 \%$ & $30 \%$ & $40 \%$ \\
\hline & PSNR & PSNR & PSNR & PSNR & PSNR & PSNR & PSNR \\
\hline None & 21.98 & 18.95 & 17.18 & 15.95 & 15.01 & 14.18 & 12.97 \\
\hline VMF & $22.95^{*}$ & $22.68^{*}$ & $22.35^{*}$ & $21.93^{*}$ & 21.70 & 21.46 & 20.77 \\
PGSF & $25.22^{*}$ & $24.00^{*}$ & $22.83^{*}$ & 22.04 & 21.51 & 20.95 & 19.66 \\
FISF & $25.29^{*}$ & $24.08^{*}$ & $23.33^{*}$ & $22.96^{*}$ & $22.39^{*}$ & $21.75^{*}$ & 20.74 \\
FIDRMC & $26.09^{*}$ & $25.48^{*}$ & $24.72^{*}$ & $24.02^{*}$ & $23.30^{*}$ & 22.86 & 21.85 \\
\hline UF & $24.17^{*}$ & $23.94^{*}$ & $23.65^{*}$ & $23.37^{*}$ & $23.07^{*}$ & $22.72^{*}$ & 21.95 \\
FRINRM & $29.12^{*}$ & $26.85^{*}$ & 25.25 & 24.55 & 23.75 & 22.82 & 20.29 \\
\hline INR & $\mathbf{3 0 . 6 4}^{*}$ & $\mathbf{2 8 . 8 8 ^ { * }}$ & $\mathbf{2 7 . 0 3}$ & $\mathbf{2 5 . 9 9}$ & $\mathbf{2 5 . 0 9}$ & $\mathbf{2 4 . 2 4}$ & $\mathbf{2 2 . 6 1}$ \\
\hline
\end{tabular}

Table 2. Some experimental results for comparison in terms of PSNR using the Boat image corrupted with different densities of random-value impulse noise

\begin{tabular}{|c|l|l|l|l|l|l|l|}
\hline Filter & $5 \%$ & $10 \%$ & $15 \%$ & $20 \%$ & $25 \%$ & $30 \%$ & $40 \%$ \\
\hline & PSNR & PSNR & PSNR & PSNR & PSNR & PSNR & PSNR \\
\hline None & 21.75 & 18.78 & 16.99 & 15.79 & 14.82 & 13.99 & 12.73 \\
\hline VMF & $30.28^{*}$ & $29.42^{*}$ & $28.20^{*}$ & $26.70^{*}$ & 26.11 & 25.46 & 23.81 \\
PGSF & $33.42^{*}$ & $30.30^{*}$ & 28.45 & 27.24 & 26.08 & 24.64 & 22.08 \\
FISF & $31.63^{*}$ & $30.14^{*}$ & $29.01^{*}$ & $27.80^{*}$ & $26.73^{*}$ & $25.16^{*}$ & 23.87 \\
FIDRMC & $34.25^{*}$ & $32.41^{*}$ & 31.00 & 29.79 & 29.05 & 27.95 & 25.80 \\
\hline UF & $33.08^{*}$ & $32.13^{*}$ & $31.32^{*}$ & $30.46^{*}$ & $29.65^{*}$ & $28.67^{*}$ & $\mathbf{2 6 . 7 9}$ \\
FRINRM & $36.80^{*}$ & 32.38 & 31.28 & 30.10 & 28.88 & 27.14 & 23.07 \\
\hline INR & $\mathbf{3 8 . 4 8}^{*}$ & $\mathbf{3 4 . 7 7}^{*}$ & $\mathbf{3 2 . 8 4}$ & $\mathbf{3 1 . 3 6}$ & $\mathbf{3 0 . 3 1}$ & $\mathbf{2 9 . 1 0}$ & 26.37 \\
\hline
\end{tabular}

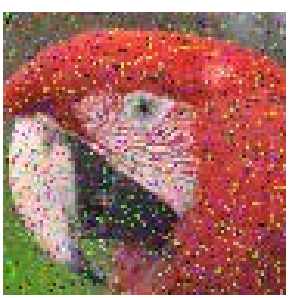

(a) $\mathrm{PSNR}=16.50$

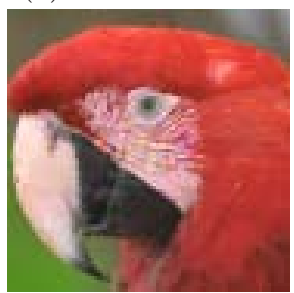

(e) $\mathrm{PSNR}=29.36$

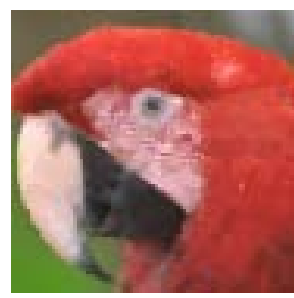

(b) $\mathrm{PSNR}=26.38^{*}$



(f) $\mathrm{PSNR}=28.01^{*}$

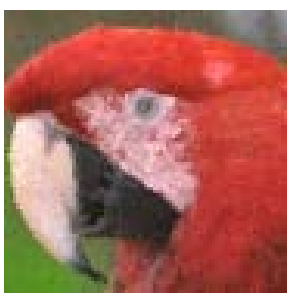

(c) $\mathrm{PSNR}=26.39$

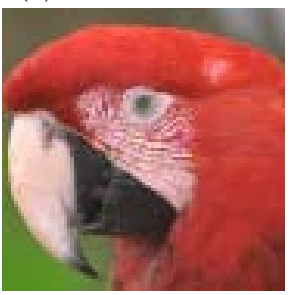

(g) PSNR $=29.99$

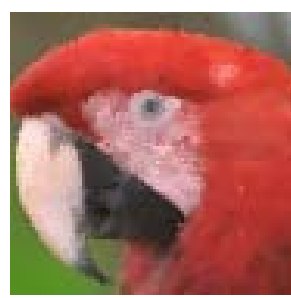

(d) $\mathrm{PSNR}=27.47^{*}$

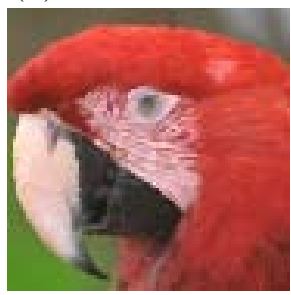

(h) $\mathrm{PSNR}=30.80$

Fig. 3. Visual comparison of filters performance. (a) Detail of Parrots image with 15\% of random-value impulse noise in each colour channel and outputs from (b) VMF, (c) PGSF, (d) FISF, (e) FIDRMC, (f) UF, (g) FRINRM and (h) INR. 
- The proposed method generally receives the best PSNR values, which indicates that the proposed method receives the best filtering capability. Other filters such as the UF, may eventually receive slightly better PSNR values however, from the visual results we illustrate that the other methods have some important disadvantages in comparison to the proposed method.

- From the images we observe the main problem of the filtering algorithms that are applied component-wise, i.e. they even introduce (impulse like) colour artefacts in heterogeneous areas like edges or fine texture areas. By processing each component separately it often happens that colour component differences were destroyed.

- The vector based approaches do not introduce artefacts but tend to cluster the noise in larger areas, as in the case of PGSF. This makes it much more difficult to reduce the remaining noise. Additionally we observe that the results from the vector based approaches tend to make the images much blurrier (smoother) than the other methods so that important image structures are destroyed.

- The best visual results were obtained by the proposed method. We observe that the proposed method reduces the noise very well, while preserving the colour information and the important image features like edges and textures.

From both the numerical and visual results we can conclude that the proposed method can be advised for reducing random-value impulse noise in colour images since it generally outperforms other state-of-the-art methods.

\section{Conclusion}

In this paper a new fuzzy filter for impulse noise reduction in colour images is presented. The main difference between the proposed method (denoted as INR) and other classical noise reduction methods is that the colour information is taken into account in a more appropriate way (i) to develop a better impulse noise reduction method and (ii) to develop a noise reduction method which reduces the noise effectively. The advantages of the proposed method are (i) it reduces random-value impulse noise (for low and high noise levels) effectively, (ii) it preserves edge sharpness and (iii) it doesn't introduce blurring artefacts or new colours artefacts in comparison to other state-of-the art methods. This method also illustrates that colour images should be treated differently than grayscale images in order to increase the visual performance. Also, this method could possibly be extended to process multispectral images produced by so many satellites.

\section{References}

1. Wang, J.H., Liu, W.J., Lin, L.D.: Histogram-Based Fuzzy Filter for Image Restoration. IEEE Transactions on Systems man and cybernetics part B.cybernetics $32(2), 230-238$ (2002) 
2. Schulte, S., Nachtegael, M., De Witte, V., Van der Weken, D., Kerre, E.E.: A Fuzzy Impulse Noise Detection and Reduction Method. IEEE Transactions on Image Processing 15(5), 1153-1162 (2006)

3. Farbiz, F., Menhaj, M.B.: A fuzzy logic control based approch for image filtering. In: Kerre, E.E., Nachtegael, M. (eds.) Fuzzy Techniques in Image Processing, vol. 52, pp. 194-221. Springer Physica Verlag, Berlin Heidelberg New York (2000)

4. Xu, H., Zhu, G., Peng, H., Wang, D.: Adaptive fuzzy switching filter for images corrupted by impulse noise. Pattern Recognition Letters 25, 1657-1663 (2004)

5. Kalaykov, I., Tolt, G.: Real-time image noise cancellation based on fuzzy similarity. In: Nachtegael, M., Van der Weken, D., Van De Ville, D., Kerre, E.E. (eds.) Fuzzy Filters for Image Processing, vol. 122, pp. 54-71. Springer Physica Verlag, Berlin Heidelberg New York (2003)

6. Schulte, S., De Witte, V., Nachtegael, M., Van der Weken, D., Kerre, E.E.: Fuzzy random impulse noise reduction method. Fuzzy Sets and Systems. In press (2007)

7. Schulte, S., De Witte, V., Nachtegael, M., Van der Weken, D., Kerre, E.E.: Fuzzy two-step filter for impulse noise reduction from color images. IEEE Transactions on Image Processing 15(11), 3567-3578 (2006)

8. David, H.A., Nagaraja, H.N.: Order Statistics, 3rd edn. Wiley, New York (2003)

9. Plataniotis, K.N., Venetsanopoulos, A.N.: Color Image Processing and Applications. Springer, Heidelberg (1998)

10. Lukac, R.: Adaptive vector median filtering. Pattern Recognition Letters 24(12), 1889-1899 (2003)

11. Astola, J., Haavisto, P., Neuvo, Y.: Vector Median Filters. IEEE Proceedings 78(4), 678-689 (1990)

12. Barni, M., Cappellini, V., Mecocci, A.: Fast vector median filter based on Euclidean norm approximation. IEEE Signal Processing Letters 1(6), 92-94 (1994)

13. Lukac, R., Plataniotis, K.N., Venetsanopoulos, A.N., Smolka, B.: A statisticallyswitched adaptive vector median filter. Journal of Intelligent and Robotic Systems 42(4), 361-391 (2005)

14. Camacho, J., Morillas, S., Latorre, P.: Efficient Impulse Noise suppression based on Statistical Confidence Limits. Journal of Imaging Science and Technology 50(5), 427-436 (2006)

15. Smolka, B., Chydzinski, A.: Fast detection and impulsive noise removal in color images. Real-Time Imaging 11(5-6), 389-402 (2005)

16. Hore, S., Qiu, B., Wu, H.R.: Improved vector filtering for color images using fuzzy noise detection. Optical Engineering 42(6), 1656-1664 (2003)

17. Morillas, S., Gregori, V., Peris-Fajarnés, G., Latorre, P.: A fast impulsive noise color image filter using fuzzy metrics. Real-Time Imaging 11(5-6), 417-428 (2005)

18. Lukac, R., Smolka, B., Martin, K., Plataniotis, K.N., Venetsanopoulos, A.N.: Vector filtering for color imaging. IEEE Signal Processing Magazine 22(1), 74-86 (2005)

19. Garnett, R., Huegerich, T., Chui, C., He, W.: A universal noise removal algorithm with an impulse detector. IEEE Transactions on Image Processing 14(11), $1747-$ 1754 (2005)

20. Kerre, E.E.: Fuzzy sets and approximate Reasoning. Xian Jiaotong University Press (1998)

21. Lee, C.C.: Fuzzy logic in control systems: fuzzy logic controller-parts 1 and 2. IEEE Transactions on Systems, Man, and Cybernetics 20(2) 404-435

22. Fodor, J.: A new look at fuzzy-connectives. Fuzzy sets and Systems 57(2), 141-148 (1993) 